\title{
Interpolation from Grid Lines: Linear, Transfinite and Weighted Method
}

\author{
Lindberg, Anne-Sofie Wessel; Jørgensen, Thomas Martini; Dahl, Vedrana Andersen
}

Published in:

Image Analysis

Link to article, DOI:

10.1007/978-3-319-59129-2 29

Publication date:

2017

Document Version

Peer reviewed version

Link back to DTU Orbit

Citation (APA):

Lindberg, A-S. W., Jørgensen, T. M., \& Dahl, V. A. (2017). Interpolation from Grid Lines: Linear, Transfinite and Weighted Method. In Image Analysis (pp. 338-349). Springer. Lecture Notes in Computer Science Vol. 10270 https://doi.org/10.1007/978-3-319-59129-2 29

\section{General rights}

Copyright and moral rights for the publications made accessible in the public portal are retained by the authors and/or other copyright owners and it is a condition of accessing publications that users recognise and abide by the legal requirements associated with these rights.

- Users may download and print one copy of any publication from the public portal for the purpose of private study or research.

- You may not further distribute the material or use it for any profit-making activity or commercial gain

- You may freely distribute the URL identifying the publication in the public portal

If you believe that this document breaches copyright please contact us providing details, and we will remove access to the work immediately and investigate your claim 


\title{
Interpolation from Grid Lines: Linear, Transfinite and Weighted Method
}

\author{
Anne-Sofie Wessel Lindberg ${ }^{(凶)}$, Thomas Martini Jørgensen, \\ and Vedrana Andersen Dahl \\ Department of Applied Mathematics and Computer Science, \\ Technical University of Denmark, 2800 Kongens Lyngby, Denmark \\ $\{$ awli, tmjq, vand $\} @ d t u . d k$
}

\begin{abstract}
When two sets of line scans are acquired orthogonal to each other, intensity values are known along the lines of a grid. To view these values as an image, intensities need to be interpolated at regularly spaced pixel positions. In this paper we evaluate three methods for interpolation from grid lines: linear, transfinite and weighted. Linear method does not preserve the known values along the grid lines. Transfinite method, known from mesh generation, preserves the known values but might cause overshoot. The weighted method, which we propose, is designed to combine the desired properties of transfinite method close to grid lines, and the stability of the linear method. We perform an extensive evaluation of the three interpolation methods across a range of upsampling rates for two data sets. Depending on the upsampling rate, we show significant difference in the performance of the three methods. We find that the transfinite interpolation works well for small upsampling rates and the proposed weighted interpolation method performs very well for all relevant upsampling rates.
\end{abstract}

Keywords: Interpolation - Image processing - Performance analysis • Line scans $\cdot$ Medical image analysis

\section{Introduction}

Scanning along a set of parallel lines is a common setting in medical imaging. The modality that motivated our investigation is optical coherence tomography (OCT) [7], well established in ophthalmology for obtaining volumetric images of the retina. Using OCT, the retina is scanned in depth $(z)$ and along a line $(x)$ with a high depth and transversal resolution, resulting in a single $x z$ cross-section of the retina (a so-called B-scan). Collecting a number of images by scanning along parallel lines results in a volumetric $x y z$ data set. Since scanning speed of the commercial systems is limited, and prolonged scanning is unpleasant for the patient, the distance between the recorded B-scans is often large compared to the transverse resolution of the B-scans. Therefore, if an $x y$ cross-section (en face) is of interest, the resolution is much coarser in $y$ direction and pixels 
are non-square. This makes it difficult to distinguish the anatomical structures, especially evident with blood vessels running parallel to scan lines.

To reveal additional anatomical structures, another OCT scan may be performed, along the lines orthogonal to the first scan. It is our goal to compute a volumetric data which combines the information from the two volumetric scans. Several problems emerge in connection to this. The eye might move in the $z$ direction during scanning, and this needs to be accounted for. Furthermore, the intensity might vary significantly between the scans and images. And most importantly, how to combine two volumetric data covering the same area, one with high resolution cross-sections in $x z$ planes, and the other in $y z$ planes?

In this paper we address the interpolation problem when merging two OCT volumes. As we have a high resolution in $z$ direction (around 5 microns), we practically sample at any height and our problem reduces to a $2 \mathrm{D}$ case. Furthermore, given a high resolution along the scan lines we ignore the discrete sampling in this direction. Therefore, our problem is image interpolation from grid lines.

The problem is illustrated in Fig. 1 (a). The information is available along the two sets of parallel lines, and it needs to be sampled at regularly spaced pixel positions.

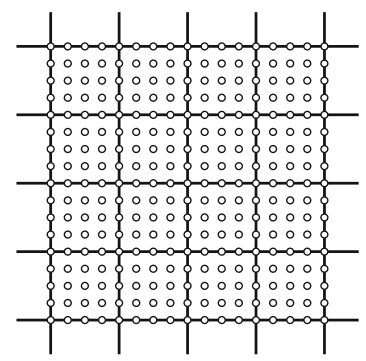

(a) problem set-up

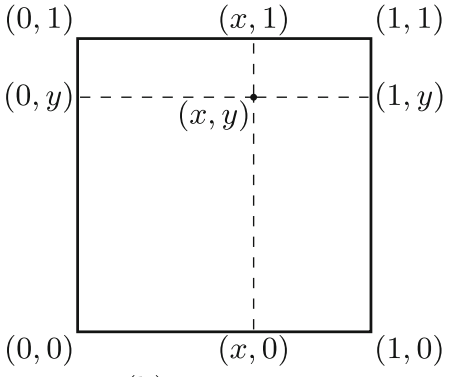

(b) one square

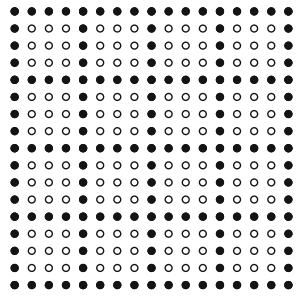

(c) testing set-up

Fig. 1. Interpolation from the grid lines. (a) two sets of scan lines with known intensities are shown in black, this need to be sampled at regularly spaced pixel positions illustrated as white dots. (b) shows one square region defined by four scan lines and its local coordinate system. (c) is a testing set-up, black are the known and white are the unknown pixels, here shown with an upsampling rate of 4 .

To the best of our knowledge, the problem of interpolating information from grid lines while preserving boundary values, has not been addressed in the context of image interpolation. In the context of mesh generation for finite element modeling a related problem is often solved using transfinite interpolation $[5,6]$, a method for constructing a smooth function over a planar domain given the values on the boundary. Transfinite interpolation has been used for solving problems where information on boundaries should be preserved. It has been used in more recently studies e.g. [12] for solving time-dependent changes of volumetric 
material properties in heterogeneous volumes and in [10] for solving elliptic boundary value Poisson problems in arbitrary shaped 2D domains.

In this work we employ the transfinite interpolation for image interpolation from grid lines, and we compare it against an approach based on linear upscaling. Furthermore, we propose a weighted interpolation which preserves the desirable properties of the transfinite and the linear method.

A quality measure for merging two OCT scans should relate to the ease of distinguishing the anatomical structures present in the volume, their sharpness and precision. While sharpness may be quantified, it is difficult to asses the precision of the interpolation. Central to our problem is that we need to determine information where it is lacking. This aspect is similar to image upscaling and single-image super-resolution approaches. Therefore, when it comes to evaluation the performance of interpolation algorithms we turn to the conventional approach $[11,16]$ which tests each method on a set of downsampled images and uses peak signal to noise ratio (PSNR) metric.

During testing, we change upsampling rates and statistically evaluate the results from the three interpolation methods. This allow us to conclude on the methods' performance and to provide guidelines for different upsampling rates.

\section{$2 \quad$ Interpolation from Grid Lines}

Figure 1 (b) illustrates our interpolation problem with the focus on a single square region defined by two pairs of neighbouring scan lines. This is a local coordinate system which we use when defining the three interpolation methods. The approach is then repeated for all squares in the image.

For a better explanation of the interpolation methods and their features, we bring an example in Fig. 2 (a). The values to be interpolated are here shown as a height above a squared domain, where we know the values at the boundary.

\subsection{Linear Interpolation}

A naive approach of combining the two scans involves linearly upsampling each scan independently and averaging the results. Over one square domain we have

$$
\begin{aligned}
L_{x}(x, y) & =(1-y) S(x, 0)+y S(x, 1), \\
L_{y}(x, y) & =(1-x) S(0, y)+x S(1, y), \\
L & =\frac{1}{2}\left(L_{x}+L_{y}\right),
\end{aligned}
$$

where $S$ are the known values along the boundary of the square domain, $L_{x}$ and $L_{y}$ are linearly upsampled boundaries in $x$ and $y$ direction, and $L$ is the interpolant which we in this context denote linear. Construction of linear interpolation is demonstrated on Fig. 2 (a)-(d).

Let us point out two properties of linear interpolation. First, every value $L(x, y)$ is a convex combination of four values from $S$. As a result, $L$ does not 


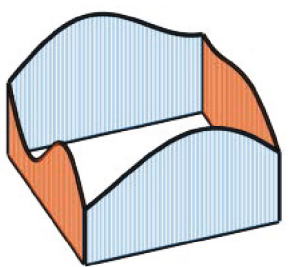

(a) input

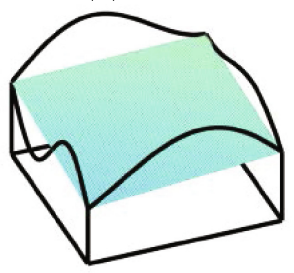

(e) $L_{x y}$

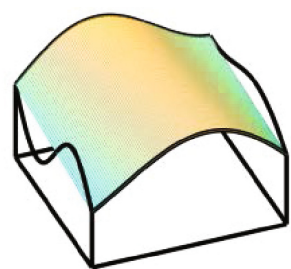

(b) $L_{x}$

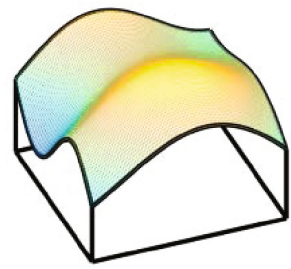

(f) $T$

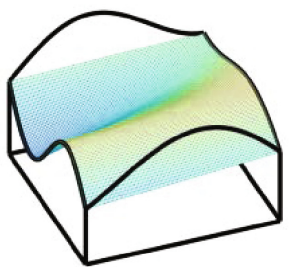

(c) $L_{y}$

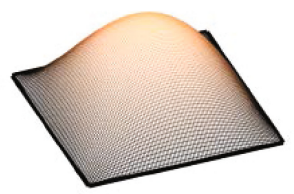

(g) $\omega$

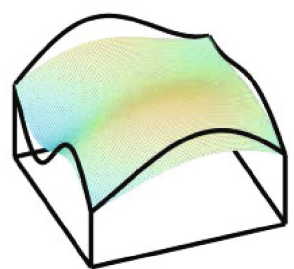

(d) $L$

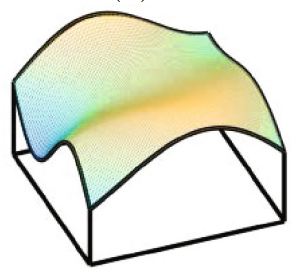

(h) $W$

Fig. 2. Interpolation over one squared domain. (a) know values from one direction in red and from the other direction in blue, (b) linear interpolation from one pair (blue) of rectangle sides, (c) linear interpolation from other pair (red) of sides, (d) mean of two linear contributions, (e) bilinear interpolation from corners, (f) transfinite interpolation, $(\mathrm{g})$ weighting scheme $(\mathrm{h})$ weighted interpolation (Color figure online).

produce undesirable overshoot. Secondly, for a point on the boundary, the underlying known data contributes only with a half of its value, the other half coming from the values at two corners. As a result, $L$ does not agree to the known data along the boundaries of the domain. Those two properties combined mean that when used on images, linear interpolation results in smeared-out appearance.

\subsection{Transfinite Interpolation}

Transfinite interpolation is used for functions given on the boundary of a domain which can be parameterized as a square. For our purposes this reduces to

$$
T=L_{x}+L_{y}-L_{x y}
$$

where

$L_{x y}(x, y)=(1-x)(1-y) S(0,0)+(1-x) y S(0,1)+x(1-y) S(1,0)+x y S(1,1)$.

Here $L_{x y}$ is the bilinear interpolant from the values at the corners of the domain, and $T$ is the final transfinite interpolant. Those are shown in Fig. 2 (e) and (f).

The most important property of the transfinite interpolation is that it preserves the known values at the boundary of the domain. To perceive how this property is achieved by the construction of $T$, note in Fig. 2 that at the boundary of the domain, $L_{x y}$ differs from the known values exactly twice as much as $L$ does. 
Second important property is that $T$ may overshoot. This is due to the negative term in the expression. All inside points receive eight weighted contributions, and especially points close to the middle of the domain are prone to interpolation overshoot, also visible in Fig. 2 (f).

\subsection{Weighted Interpolation}

Transfinite interpolation has the desired properties (preservation of the known data) at the boundary of the square domain while the undesired properties (overshoot) are inside the domain. Linear interpolation does not overshoot, but has issues at the boundary. To combine the good properties of both methods we propose smoothly blending the linear and the transfinite interpolation. We construct a blending function which is 0 at the boundary of the square domain

$$
\omega(x, y)=2^{4} x(1-x) y(1-y) .
$$

The constant $2^{4}$ is chosen such that $\omega(0.5,0.5)=1$.

We define our novel interpolation, which we denote weighted, as

$$
W=\omega L+(1-\omega) T \text {. }
$$

This also evaluates to

$$
W=(2-\omega) L-(1-\omega) L_{x y}
$$

The blending function and the weighted interpolation are shown on Fig. 2 (g) and $(\mathrm{h})$. The illustrated example confirms desirable properties of the weighted interpolation. Like transfinite, the weighted interpolant matches the exact values at the boundaries of the domain. However, thanks to blending with the linear interpolant, the overshoot from inside of the domain is reduced. Finally, smooth blending function maintains a smooth appearance of the interpolant.

\section{Evaluation of Interpolation Methods}

Both objective and subjective tests are used [4] for evaluating interpolation methods. Subjective tests measure a perceived image quality, while objective tests use a defined metrics for quantifying image quality or interpolation error. The choice of the tests and the quality measures depends on the intended use.

For our motivating example, interpolation from grid lines is a step towards merging two OCT line scans. We plan to use the merged volume for automatic detection of anatomical structures and quantification of abnormalities in the eye. In this upcoming work we will evaluate the three interpolation methods in terms of the detection and quantification results.

In the work presented here, we bring a more meticulous and general evaluation of the interpolation methods based on measuring interpolation error for a specific upsampling rates. The ground truth is constructed by downsampling 
an image, which is then upsampled using the three methods, and the results are compared against the original image. For downsampling, we keep image columns and rows at a certain distance, which corresponds to upsampling rate $s$. See Fig. 1 (c) for our testing set-up.

For a certain upsampling rate, the fraction of the unknown pixel is

$$
u=\frac{(s-1)^{2}}{s^{2}} .
$$

For example, when $s=2$ we keep every second row and every second column, and fraction of unknown pixels is only 0.25 .

To demonstrate the properties of the interpolation methods, we conduct tests for upsampling rates from 2 to 30. However, the high upsampling rates (above 10) are of limited practical value due to high degeneration of image quality.

\subsection{Data Sets}

We evaluate the three interpolation methods on two data sets. The first contains 200 images from the Berkley Segmentation data set [9], which is widely used for evaluations of image upscaling and super-resolution algorithms [2]. The images depict scenes from nature such as landscapes, people and animals, covering a wide range of image patterns at all scales. We converted all images into grayscale with an intensity range between 0 and 1 prior to processing.

The second data set is ophthalmologic data in form of 72 funduscopies. Fundoscopy is an imaging technique for examination of fundus obtained using a light source and a ophthalmoscope. This was chosen because of image content which is similar to OCT scans, and will allow us to assess the performance of the interpolation methods in a setting which resembles to our application. Figure 3 shows some examples of the funduscopies.
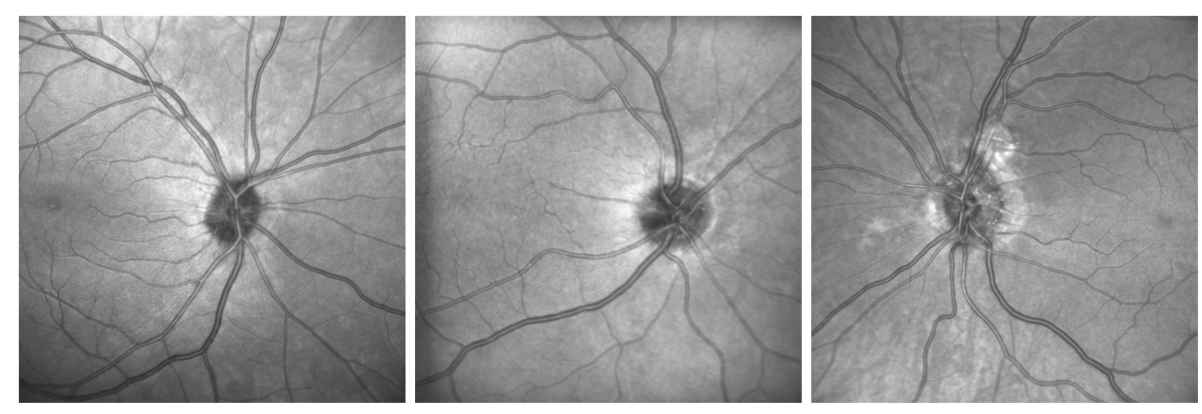

Fig. 3. Three images from the fundoscopy data set. Images depict anatomical structures at the fundus of the eye. 


\subsection{Performance Measurement}

The interpolation quality is assessed by the pixelwise difference between the ground truth image and the interpolated image. The interpolation error can be evaluated as the Root Mean Square Error (RMSE) which is defined as [8]

$$
\operatorname{RMSE}=\sqrt{\frac{1}{N} \sum_{i=1}^{N}(\hat{I}(i)-I(i))^{2}}
$$

where summation runs over all pixels $i$ from the original image $I$ and the interpolated image $\hat{I}$.

More often the Peak Signal-to-Noise Ratio (PSNR) is used [16] as an interpolation quality measure. The PSNR is measured in $\mathrm{dB}$ and defined as [14]

$$
\mathrm{PSNR}=20 \log _{10}\left(\frac{\mathrm{MAX}_{\mathrm{I}}}{\mathrm{RMSE}}\right)
$$

where $\mathrm{MAX}_{\mathrm{I}}$ is the maximum pixel intensity value, in our case 1 .

\section{Results}

First, we present some results from the interpolations for upsampling rate 3 and 6. Second, we present the performance of each interpolation method for upsampling rates varying between 2 and 30. Last, we present the results from a statistical analysis of the methods' performance.

\subsection{Interpolated Results}

The differences in performance of the three interpolation methods are subtle, and to visualize the results we bring a small detail of an image from the Berkley segmentation data set in Fig. 4 (a), and we also show the grid lines for upsampling rate 3 and 6 in Fig. 4 (b) and (c). The interpolated results for this image, the two sets of grid lines and the three interpolation methods are shown on Fig. 5. We also bring the pixelwise error between the interpolated images and the original image. It can be seen that the error is zero along the grid lines for the transfinite and the weighted interpolation, while this is not the case for the linear interpolation. Furthermore, for all methods, the interpolation quality in form of the PSNR decreases when the upsampling rate is increased. For this example, the weighted interpolation outperforms the transfinite when using upsampling rate 6 .

\subsection{Performance Analysis}

Figure 6 shows the PSNR values for the three interpolation methods for 6 randomly chosen images from the Berkley segmentation data set, interpolated with upsampling rate 6 . We see a big variance in performance across the images, 


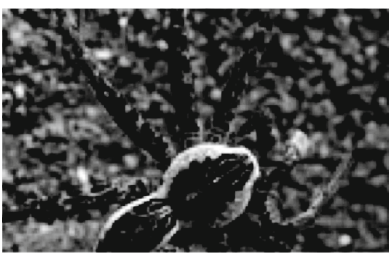

(a) detail

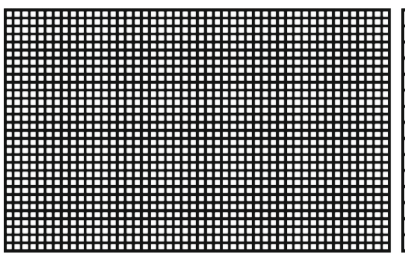

(b) sampling 3

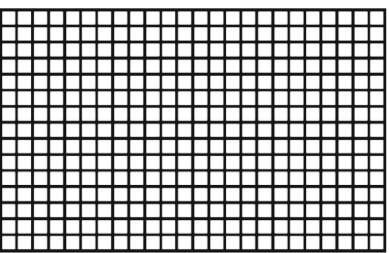

(c) sampling 6

Fig. 4. Testing example. (a) zoom on a detail in one of the images from the Berkley data set. (b) and (c) are grid lines with an upsampling rate of 3 and 6 .

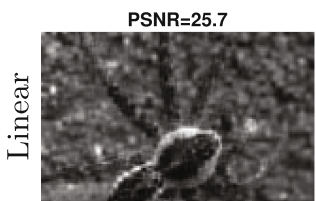

PSNR=27.4

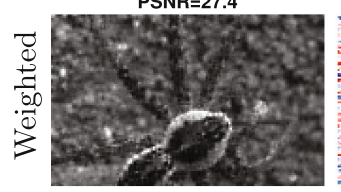

PSNR=27.4

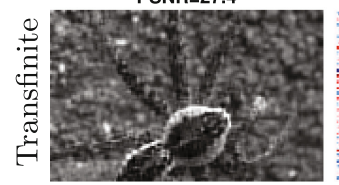

(a) sampling 3

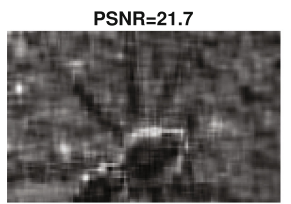

PSNR=22.3
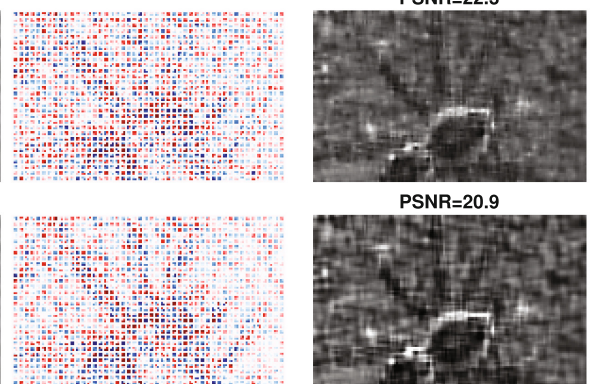

PSNR=20.9

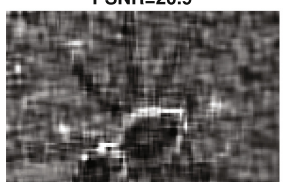

(b) sampling 6
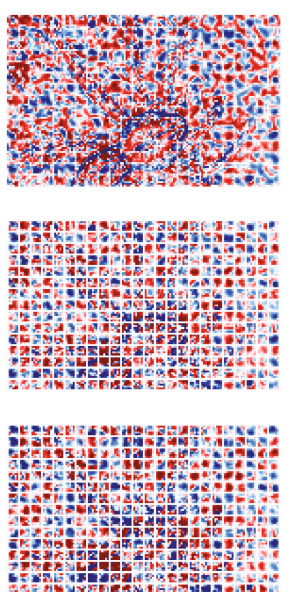

Fig. 5. The interpolated results for the detail and the grid lines shown on Fig. 4. The interpolation methods are presented with linear on top, weighted in the middle and transfinite in the bottom row. Columns (a) and (b) bring the results for an upsampling rate of 3 and 6 . The PSNR value for each interpolated image is listed above it. Next to each interpolated image is the pixelwise difference between the interpolated image and the original image, with red/blue color indicating positive/negative difference, and white indicating zero difference. (Color figure online)

compared to relatively small variance between the three interpolation methods. However, weighted interpolation obtains the best performance for 5 out of 6 images, while transfinite method is best for the last image. Linear method is not the best for any of the images, but is still superior to transfinite in 4 out of 6 images.

To evaluate an overall performance of the interpolation methods, we computed the mean PSNR for the whole Berkley segmentation data set, for each interpolation method and for a range of upsampling rates. We conducted a similar experiment for the Fundoscopy data set. Figure 7 shows a plot of the obtained values with upsampling rates varying between 2 and 19 . We notice the 
same performance pattern for both data sets. The transfinite method has the largest mean PSNR for smallest upsampling rates, while linear method has the largest mean PSNR for highest upsampling rates. In the interval around the point where transfinite and linear method cross, the weighted method achieves the highest mean PSNR.

We conducted experiments for upsampling rate up to 30 , and we confirm that the linear method achieves best mean PSNR for high upsampling rates. We find this being of limited practical value, as for upsampling rates higher than 18 we interpolate over $89 \%$ of the pixels in the image.

As already shown in Fig. 6 the variance of performances is big between the images and small between the methods. To confirm our findings presented in Fig. 7, we performed a statistical test of the interpolation performance measured by the PSNR value. We set up a regression model for correlation between the PSNR value and the categorical variables for image and for method, for each sampling rate. F-values indicated that the method is the main descriptor. Moreover, we found that a significant difference between the three methods exists. Therefore, we tested the methods pairwise to check for difference between them at each upsampling rate and moreover, to find out which method performs best. The results are listed in Table 1(a) for the Berkley data set and in Table 1(b) for the Fundoscopy data set. The results show similar trend, and we use notation $a / b$ when referring to the two data sets. It is seen that the transfinite interpolation
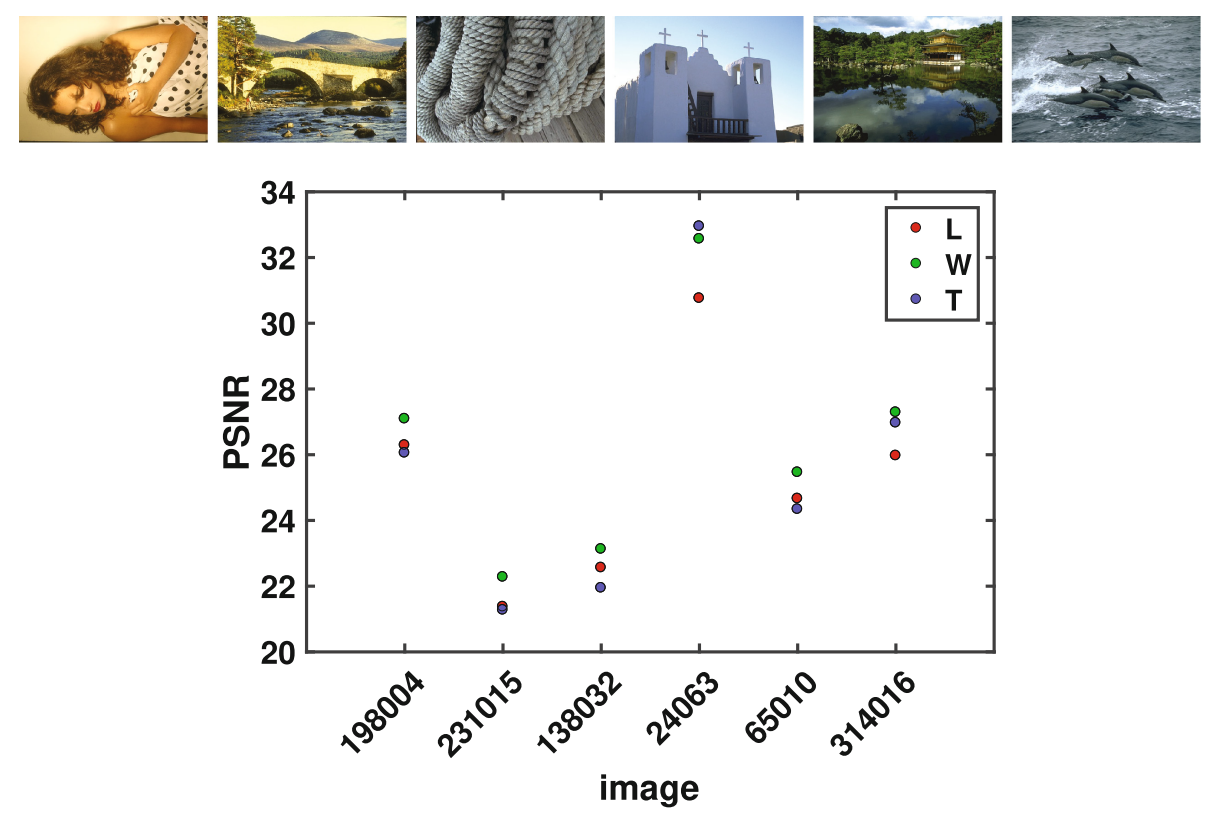

Fig. 6. A set of 6 randomly chosen images from the Berkley segmentation data set, and the resulting PSNR for three interpolation methods, linear (L), weighted (W) and transfinite $(\mathrm{T})$. Upsampling rate is 6 . 


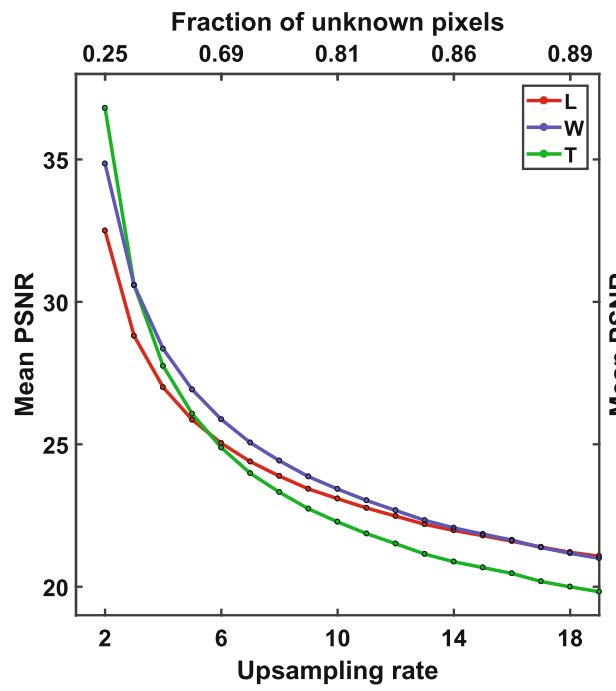

(a) Berkley data set

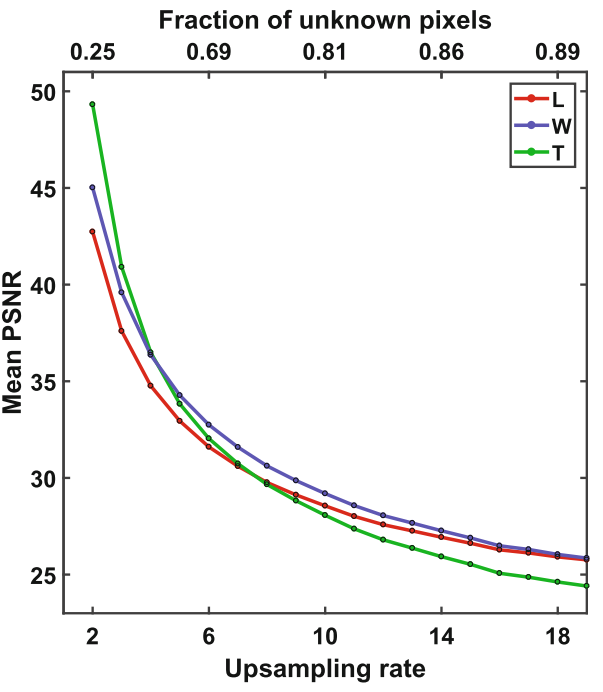

(b) Fundoscopy data set

Fig. 7. Comparison of the linear (L), weighted (W) and transfinite (T) interpolation. The mean PSNR value for (a) Berkley and (b) Fundoscopy data set at different upsampling rate. The second $\mathrm{x}$-axis indicates the fraction of unknown pixels.

Table 1. Results from statistical analysis of interpolation performance for three methods at different upsampling rates, for Berkley data set (a) and Fundoscopy data set (b). The methods are linear $(\mathrm{L})$, weighted $(\mathrm{W})$ and transfinite $(\mathrm{T})$, and upsampling rates are shown in intervals between 2 and 30. Number 1 indicates the method that performs best for a given upsampling rate, while 3 indicates the method that performs worst. The star indicates that no significant difference was found between the two methods for the given upsampling rate.

(a) Berkley data set

\begin{tabular}{|c|ccccccc|}
\cline { 2 - 8 } \multicolumn{1}{c|}{} & 2 & 3 & $4-5$ & 6 & $7-14$ & $15-20$ & $21-30$ \\
\hline $\mathrm{L}$ & 3 & 3 & 3 & $2^{*}$ & 2 & $\mathbf{1}^{*}$ & $\mathbf{1}$ \\
$\mathrm{W}$ & 2 & $\mathbf{1}^{*}$ & $\mathbf{1}$ & $\mathbf{1}$ & $\mathbf{1}$ & $\mathbf{1}^{*}$ & 2 \\
$\mathrm{~T}$ & $\mathbf{1}$ & $\mathbf{1}^{*}$ & 2 & $2^{*}$ & 3 & 3 & 3 \\
\hline
\end{tabular}

(b) Fundoscopy data set

\begin{tabular}{|c|ccccccc|}
\cline { 2 - 8 } \multicolumn{1}{l|}{} & $2-3$ & 4 & $5-6$ & $7-8$ & $9-19$ & $20-22$ & $23-30$ \\
\hline $\mathrm{L}$ & 3 & 3 & 3 & $2^{*}$ & 2 & $\mathbf{1}^{*}$ & $\mathbf{1}$ \\
$\mathrm{W}$ & 2 & $\mathbf{1}^{*}$ & $\mathbf{1}$ & $\mathbf{1}$ & $\mathbf{1}$ & $\mathbf{1}^{*}$ & 2 \\
$\mathrm{~T}$ & $\mathbf{1}$ & $\mathbf{1}^{*}$ & 2 & $2^{*}$ & 3 & 3 & 3 \\
\hline
\end{tabular}

performs best for upsampling rates below $3 / 4$ and the weighted interpolation performs best for sampling rates above $4 / 5$ and below $15 / 20$. The linear method performs best for upsampling rates above $20 / 22$.

\section{Discussion}

Our experiments and the statistical evaluation of the three interpolations are in alignment with the previously demonstrated properties of the methods. Prior to experiments, we knew that the transfinite methods performs best close to the 
grid lines containing known information, while the linear method performs best in areas further away from the grid lines. Therefore we expected the transfinite method to achieve superior results for small upsampling rates where grid lines cover a large fraction of the image. Our results confirm this hypothesis. Likewise, we show that linear method is superior at high upsampling rates.

We designed the weighted method to combine the good properties of the linear and transfinite method. Our results confirm that weighted method has superior performance for a large interval of upsampling rates, and especially where the transfinite and linear method perform equally badly. This happens at upsampling rate of $6 / 8$ and the interval where weighted method is superior extends from $4 / 5$ to $14 / 19$. For our application of merging OCT images we are aiming at upsampling ratio between 5 and 10 and these results indicate that weighted interpolation should be used.

It is important to note that image quality measured as PSNR not directly correspond to high quality of the interpolation. Further investigation that measures image quality in terms of sharpness should be performed for finding the most suitable method. Likewise, if the images are to be used for visual inspection, a perceived quality of the images should be measured.

The three interpolation methods presented here only use the known intensity information along the grid lines. We would expect the performance to improve significantly if a prior knowledge about the appearance of images is incorporated in the method. A significant work in this line has been conducted for single-image super resolution [3] or image inpainting [1], for example using image patches [15] and sparse representation [13]. We believe that those methods might be adapted to solve the problem of interpolation from the grid lines.

\section{Conclusion}

In conclusion, the contribution of our present work is twofold. First, we introduce the problem of interpolation from grid lines and suggest three possible solutions: a linear, a transfinite and a weighed interpolation. Secondly, we provide systematically test the three methods and conclude that transfinite method is superior for very small upsampling rates, while weighted method should be considered for a broad range of upsampling rates.

\section{References}

1. Bertalmio, M., Sapiro, G., Caselles, V., Ballester, C.: Image inpainting. In: Proceedings of the 27th Annual Conference on Computer Graphics and Interactive Techniques, pp. 417-424 (2000)

2. Dong, W., Zhang, L., Shi, G., Wu, X.: Image deblurring and super-resolution by adaptive sparse domain selection and adaptive regularization. IEEE Trans. Image Process. 20(7), 1838-1857 (2011)

3. Elad, M., Feuer, A.: Restoration of a single superresolution image from several blurred, noisy, and undersampled measured images. IEEE Trans. Image Process. 6(12), 1646-1658 (1997) 
4. Giachetti, A., Asuni, N.: Real-time artifact-free image upscaling. IEEE Trans. Image Process. 20(10), 2760-2768 (2011)

5. Gordon, W.J., Hall, C.A.: Construction of curvilinear co-ordinate systems and applications to mesh generation. Int. J. Numer. Methods Eng. 7(4), 461-477 (1973)

6. Gordon, W.J., Hall, C.A.: Transfinite element methods: blending-function interpolation over arbitrary curved element domains. Numer. Math. 21(2), 109-129 (1973)

7. Huang, D., Swanson, E.A., Lin, C.P., Schuman, J.S., Stinson, W.G., Chang, W., Hee, M.R., Flotte, T., Gregory, K., Puliafito, C.A., Fujimoto, J.G.: Optical coherence tomography. Science 254(5035), 1178 (1991)

8. Loia, V., Sessa, S.: Fuzzy relation equations for coding/decoding processes of images and videos. Inf. Sci. 171(1-3), 145-172 (2005)

9. Martin, D., Fowlkes, C., Tal, D., Malik, J.: A database of human segmented natural images and its application to evaluating segmentation algorithms and measuring ecological statistics. In: Proceedings of the 8th International Conference in Computer Vision, vol. 2, pp. 416-423, July 2001

10. Provatidis, C.G.: Solution of two-dimensional Poisson problems in quadrilateral domains using transfinite coons interpolation. Commun. Numer. Methods Eng. 20(7), 521-533 (2004)

11. Romano, Y., Protter, M., Elad, M.: Single image interpolation via adaptive nonlocal sparsity-based modeling. IEEE Trans. Image Process. 23(7), 3085-3098 (2014)

12. Sanchez, M., Fryazinov, O., Adzhiev, V., Comninos, P., Pasko, A.: Space-time transfinite interpolation of volumetric material properties. Trans. Vis. Comput. Graph. 21(2), 278-288 (2015)

13. Shen, B., Hu, W., Zhang, Y., Zhang, Y.J.: Image inpainting via sparse representation. In: Proceedings of the IEEE International Conference on Acoustics, Speech, and Signal Processing, pp. 697-700, April 2009

14. Sung, M.M., Kim, H.J., Kim, E.K., Kwak, J.Y., Yoo, J.K., Yoo, H.S.: Clinical evaluation of JPEG2000 compression for digital mammography. Nucl. Sci. 49(3), 827-832 (2002)

15. Xu, Z., Sun, J.: Image inpainting by patch propagation using patch sparsity. Trans. Image Process. 19(5), 1153-1165 (2010)

16. Yang, C.-Y., Ma, C., Yang, M.-H.: Single-image super-resolution: a benchmark. In: Fleet, D., Pajdla, T., Schiele, B., Tuytelaars, T. (eds.) ECCV 2014. LNCS, vol. 8692, pp. 372-386. Springer, Cham (2014). doi:10.1007/978-3-319-10593-2_25 